NASA/TM-2012-216042

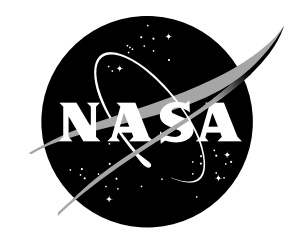

\title{
Dual-Pulse Pulse Position Modulation (DPPM) for Deep-Space Optical Communications: Performance and Practicality Analysis
}

Jing Li

Lehigh University, Bethlehem, Pennsylvania

Alan Hylton, James Budinger, Jennifer Nappier, Joseph Downey, and Daniel Raible

Glenn Research Center, Cleveland, Ohio 


\section{NASA STI Program . . . in Profile}

Since its founding, NASA has been dedicated to the advancement of aeronautics and space science. The NASA Scientific and Technical Information (STI) program plays a key part in helping NASA maintain this important role.

The NASA STI Program operates under the auspices of the Agency Chief Information Officer. It collects, organizes, provides for archiving, and disseminates NASA's STI. The NASA STI program provides access to the NASA Aeronautics and Space Database and its public interface, the NASA Technical Reports Server, thus providing one of the largest collections of aeronautical and space science STI in the world. Results are published in both non-NASA channels and by NASA in the NASA STI Report Series, which includes the following report types:

- TECHNICAL PUBLICATION. Reports of completed research or a major significant phase of research that present the results of NASA programs and include extensive data or theoretical analysis. Includes compilations of significant scientific and technical data and information deemed to be of continuing reference value. NASA counterpart of peer-reviewed formal professional papers but has less stringent limitations on manuscript length and extent of graphic presentations.

- TECHNICAL MEMORANDUM. Scientific and technical findings that are preliminary or of specialized interest, e.g., quick release reports, working papers, and bibliographies that contain minimal annotation. Does not contain extensive analysis.

- CONTRACTOR REPORT. Scientific and technical findings by NASA-sponsored contractors and grantees.
- CONFERENCE PUBLICATION. Collected papers from scientific and technical conferences, symposia, seminars, or other meetings sponsored or cosponsored by NASA.

- SPECIAL PUBLICATION. Scientific, technical, or historical information from NASA programs, projects, and missions, often concerned with subjects having substantial public interest.

- TECHNICAL TRANSLATION. Englishlanguage translations of foreign scientific and technical material pertinent to NASA's mission.

Specialized services also include creating custom thesauri, building customized databases, organizing and publishing research results.

For more information about the NASA STI program, see the following:

- Access the NASA STI program home page at http://www.sti.nasa.gov

- E-mail your question to help@sti.nasa.gov

- Fax your question to the NASA STI Information Desk at 443-757-5803

- Phone the NASA STI Information Desk at 443-757-5802

- Write to: STI Information Desk NASA Center for AeroSpace Information 7115 Standard Drive Hanover, MD 21076-1320 
NASA/TM-2012-216042

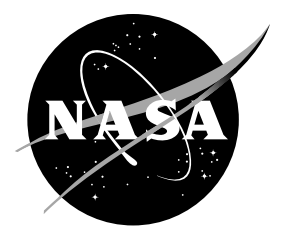

\section{Dual-Pulse Pulse Position Modulation (DPPM) for Deep-Space Optical Communications: Performance and Practicality Analysis}

Jing Li

Lehigh University, Bethlehem, Pennsylvania

Alan Hylton, James Budinger, Jennifer Nappier, Joseph Downey, and Daniel Raible

Glenn Research Center, Cleveland, Ohio

National Aeronautics and

Space Administration

Glenn Research Center

Cleveland, Ohio 44135 
Trade names and trademarks are used in this report for identification only. Their usage does not constitute an official endorsement, either expressed or implied, by the National Aeronautics and Space Administration.

Level of Review: This material has been technically reviewed by technical management.

Available from

NASA Center for Aerospace Information 7115 Standard Drive

Hanover, MD 21076-1320
National Technical Information Service 5301 Shawnee Road Alexandria, VA 22312

Available electronically at http://www.sti.nasa.gov 


\title{
Dual-Pulse Pulse Position Modulation (DPPM) for Deep-Space Optical Communications: Performance and Practicality Analysis
}

\author{
Jing Li \\ Lehigh University \\ Bethlehem, Pennsylvania 18015

\begin{abstract}
Alan Hylton, James Budinger, Jennifer Nappier, Joseph Downey, and Daniel Raible
National Aeronautics and Space Administration

Glenn Research Center

Cleveland, Ohio 44135
\end{abstract}

\begin{abstract}
Due to its simplicity and robustness against wavefront distortion, pulse position modulation (PPM) with photon counting detector has been seriously considered for long-haul optical wireless systems. This paper evaluates the dual-pulse case and compares it with the conventional single-pulse case. Analytical expressions for symbol error rate and bit error rate are first derived and numerically evaluated, for the strong, negativeexponential turbulent atmosphere; and bandwidth efficiency and throughput are subsequently assessed. It is shown that, under a set of practical constraints including pulse width and pulse repetition frequency (PRF), dual-pulse PPM enables a better channel utilization and hence a higher throughput than it singlepulse counterpart. This result is new and different from the previous idealistic studies that showed multi-pulse PPM provided no essential information-theoretic gains than single-pulse PPM.
\end{abstract}

\section{INTRODUCTION}

Optical wireless communications, or, free-space optics (FSO), has been widely used in inter-satellite and deep space communications, as well as in roof-top networks and as a viable solution for the "last mile" problem. A number of viable modulation schemes have been proposed for optical wireless systems, including on-off keying (OOK), pulse position modulation (PPM), differential phase shift keying (DPSK), and even pulse width modulation (PWM). Among them, PPM with direct detection involving photon-counting receiver is regarded by many as highly advantageous, especially for longhaul transmission such as inter-satellite, lunar, and deep-space communications.

From the information-theoretic perspective, it has long been known that under peak and average power constraints, restricting the modulation to a binary, slotted scheme results in only negligible capacity loss [3]. More recent analysis shows that, under the peak and average power constraints typical of a deep-space link, restricting the modulation to PPM results in near-capacity performance also [10].

From the practical perspective, PPM signals are easy and fast to modulate. They also allow for efficient photo-counting receivers such as avalanche photodiode (APD) detectors. In general, as optical signals transmit though a turbulent atmosphere, they encounter a large amount of wavefront distortion in amplitude and especially in phase. To correct phase distortion is difficult, but is a must-have if single-mode operation or diffraction-limited focusing is required. Since a photon counting receiver does not need to amplify the optical signal, the constraint for single-mode operation is relaxed, and, so long as the photon-counting detector placed in the receiver focal plane has adequate detection area, the second constraint is also relaxed. Hence, PPM with photon counting receivers is relatively robust against wavefront distortion due to atmospheric turbulence or scintillation. Additionally, PPM is also particularly desirable for lunar or deep-space communication systems, since the superb sensitivity of the downlink photocounting receiver can effectively reduce the required poweraperture product needed to operate the transmitter on the satellite, which in term reduces the size, weight, and power of the satellite.

There are two types of PPM schemes.

(1) In a conventional $M$-slot (single-pulse) PPM, a PPM symbol interval is divided into $M$ (even) time slots, and a block of $m=\log _{2} M$ binary bits are mapped to one of the $M$ time slots, signified by an optical pulse in this very time slot. The rate of the modulation, defined as the number of output symbols vs the incoming bits is given as $M / \log _{2} M=2^{m} / m$.

(2) To improve the data rate and throughput, multi-pulse PPM (MPPM) [1], also known as combinatorial PPM [2], has been developed as an extension to the single-pulse PPM. Since two or more pulses are allowed to convey information in each $M$-slot symbol, there are potentially $M_{l} \triangleq\left(\begin{array}{c}M \\ l\end{array}\right)$ possible $l$ pulse combinations, leading to a payload of up to $\left\lfloor\log _{2}\left(M_{l}\right)\right\rfloor$ bits per modulated symbol. Although the concept of MPPM dates back to the late eighties (e.g. [1], [2]), the scheme has recently received a revived interest, spurred in part by the ever increasing data rate demand of today's lunar and deep-space communication systems (which now target a downlink data rate of $600 \mathrm{Mbps}$ to $1 \mathrm{Gbps}$ ), and in part by the emerging technology of visible light communication (in which MPPM can be used to, for example, simultaneously control brightness and communicate data [5]).

For a propagation distance of less than $100 \mathrm{~m}$, the optical signals tend to experience relatively weak turbulence, which may be modeled as a log-normal distribution with good accuracy. This generally corresponds to a scintillation index in the range of $[0,0.75]$. As the communication range increases, 
the accuracy of the log-normal distribution quickly fades. Over the years, several statistical models have been developed to describe FSO channels under a variety of atmospheric turbulence conditions, including $\mathrm{K}$ distribution and I-K distribution [6], log-normal Rician distribution [7], gamma-gamma distribution [8], [9], and the negative-exponential distribution [10]. Although the gamma-gamma channel model, based on the modified Rytov theory, captures a fairly wide range of turbulent conditions, the negative-exponential channel model remains one of the most popular models for strong scintillation (when scintillation index is 1), due to its good accuracy and analytical tractability.

The performance of single-pulse PPM, especially at relatively low order such as $M=2$ and 4 , have been extensively studied in both weak and strong atmospheric scintillation. Noteworthy studies include, for example, uncoded $M$-ary PPM over log-normal and negative-exponential channels [10], ReedSolomon (RS) coded $M$-ary PPM over log-normal channels [11], Reed-Solomon (RS) coded $M$-ary PPM over negativeexponential channels [12]. In comparison, the performance of multi-pulse PPM is much less studied. Not considering atmospheric scintillation, [4] performed a compressive study of the error rate performance of dual-pulse PPM (DPPM) by evaluating a pure Poisson counting process. However, the performance of a scintillated DPPM FSO system has not received adequate attention. This paper analyzes the error rate performance of uncoded DPPM, as well as its bandwidth efficiency and throughput, with comparison to single-pulse PPM. Targeting lunar and deep space communications, we consider strong atmospheric scintillation that is modeled as the negative-exponential distribution.

Our main contributions include:

(1) We developer the analytical expressions for the symbol error rate (SER) and the bit error rate (BER) of dual-pulse PPM (and single-pulse PPM) over negative-exponential FSO channels. We show that, for the same $M=2^{m}$-slot symbol, DPPM incurs very marginal performance loss with but delivers $(m-2)$ bits more per symbol.

(2) Using the Z-channel model as an abstraction of the intensity-modulated directly-detected (IM/DD) FSO channel, we evaluate the channel capacity. We show that for singlepulse PPM, smaller $M$ delivers a better bandwidth efficiency (in bits/Hz/s) and performs closer to the capacity limit (and non-equal-probable OOK can achieve the capacity). We also show that, from the information-theoretic perspective, $M$-slot dual-pulse PPM is the equivalence to $M / 2$-slot single-pulse PPM, which suggests little need to adopt dual-pulse PPM.

(3) We further evaluate the system by considering the practicality issues. The clock frequency sets a limit on the minimum slot width, and the pulse repetition frequency (PRF) sets a constraint on the minimum guard-time (between two PPM symbols). Hence, in single-pulse PPM as well in dualpulse PPM, a smaller $M$ does not necessarily lead to a better bandwidth efficiency. For a given set of realistic constraints, optimal values of $M$ can be derived to maximize the throughput of either case. In general, carefully-designed dual-pulse
PPM can expect to reduce the amount of guard-time, improve the channel utilization, and hence outperform single-pulse PPM. For specific (realistic) system profiles, it is possible for a carefully-designed dual-pulse PPM scheme to be $20 \%$ more bandwidth efficient than the optimal single-pulse PPM, with the same energy efficiency.

\section{System Model}

Consider an FSO communication system that employs intensity modulation with direct detection. Light emitting diodes (LED) or laser diodes (LD) are used as transmitters, and avalanche photodiodes (APD) involving photon counting is used as the receiver.

As a laser beam shoots through the turbulent atmosphere, the intensity of the optical field experiences random fluctuations, commonly known as atmospheric scintillation or atmospheric turbulence. The scintillation index is a rather complicated function, which is mostly governed by the fluctuation of the index of fraction (due to temperature variations) and the propagation distance, but is also related to the beam parameters, and the heights of the transmitter and receiver.

\section{A. Strong Atmospheric Scintillation}

The log-normal fading model is rather accurate for clear-air scintillation with a fairly short transmit range, but its accuracy fades when the transmit distance increases beyond $100 \mathrm{~m}$ and when the scintillation indexes exceeds 0.75 . Researchers have shown that strong atmospheric scintillation may be modeled as negative exponential distribution, which corresponds to a scintillation index of 1 .

The notations used in the discussion are listed as follows.

\begin{tabular}{c|l}
\hline$M$ & the number of slots per PPM symbol, $m=$ \\
$T_{s}$ & $\log _{2} M$ \\
$\tau$ & the PPM symbol duration \\
$T_{\theta}$ & the PPM slot time \\
$K_{s}$ & the PPM symbol guard time \\
$K_{b}$ & the background-noise/radiation photon count per \\
& PPM "space" slot \\
$\Gamma\left(K_{s}\right)$ & the equivalent signal-to-noise ratio (SNR) for the \\
& optical detection process \\
\hline$P_{e}$ & symbol error rate for single-pulse PPM (i.e. \\
& misdetecting a mark) \\
$P_{e}^{d p}$ & symbol error rate for dual-pulse PPM \\
$P_{b}$ & bit error rate for single-pulse PPM \\
$P_{b}^{d p}$ & bit error rate for dual-pulse PPM \\
\hline
\end{tabular}

We have the following additional comments:

(1) In an ideal situation, the slot time $\tau$ relates to the symbol duration $T_{s}$ by $T_{s}=M \tau$, where a slot may be either a signal slot ("mark") or an empty slot ("space"). In practice, however, there may be constraints on pulse repetition frequency (PRF) due to the physical limitation of the laser diodes, such that two pulses may not be shoot immediately back to back. This results in a minimal time interval requirement between any two consecutive marks, which necessitates the allocation of 
guard time $T_{g}$ between two symbols. Hence, $T_{s}=M \tau+T_{g}$. In practice, the guard time $T_{g}$ may be as small as one PPM slot interval, or as large as $25 \%$ of the $M$-PPM slots: $\tau \leq$ $T_{g} \leq M \tau / 4$.

The APD direct-detection receiver performs a photodetection process, which can be modeled fairly accurately as a Poisson point process over $M$ distinct and disjoint counting intervals ( $M$ PPM slots). The probability for each count component is Poisson distribution with an average value $K_{b}=\lambda_{b} \tau$ for the space slots and $K_{s}+K_{b}=\left(\lambda_{s}+\lambda_{b}\right) \tau$ for the mark slots. For simplicity, the arrival rates $\lambda_{s}$ and $\lambda_{b}$ are assumed constants over a PPM slot. $\lambda_{s}$ is related to several factors, including the quantum efficiency of the detector $\eta$, the intensity of the received optical field $I$, the detector area $A_{d}$, the wavelength of the light $v$, and Planck's constant: $\lambda_{s}=\frac{\eta A_{d} I}{h v}$.

(2) In a perfect "clear-air free-space" case, such as intersatellite communication outside of the earth atmosphere, $I$ and $\lambda_{s}$ remains a constant throughput a transmission session, and hence $K_{s}$ follows a Poisson distribution. However, when the signal travels through (part of) the atmosphere, the optical intensity $I$ is no longer invariant, but becomes a random variable. Specifically, under strong atmospheric scintillation, $I$ may be well-approximated by a negative exponential distribution, and consequently, the photon count per PPM slot also follows a negative exponential distribution, whose pdf is given by

$$
f\left(K_{s}\right)= \begin{cases}\frac{1}{\lambda_{s} \tau} \exp \left(-\frac{K_{s}}{\lambda_{s} \tau}\right), & \text { for } K_{s} \geq 0, \\ 0, & \text { otherwise. }\end{cases}
$$

where $\lambda_{s} \tau=E\left[K_{s}\right]$ is the average photon count per PPM mark slot.

(3) The equivalent $\operatorname{SNR} \Gamma\left(K_{s}\right)$ is given by

$$
\Gamma\left(K_{s}\right)=\frac{K_{s}^{2}}{F K_{s}+K_{n}}
$$

Where $K_{n}$ denotes the overall impact of thermal noise and background photon radiation, and is a function of the receiver temperature (in degrees Kelvin) $T^{o}$, the Boltzmann constant $\kappa$, the average APD gain $g$, the noise factor of the APD $F$, the APD load resistance $R_{l}$, and the PPM slot duration $\tau$ and the background photon count $K_{b}$ :

$$
K_{n}=2 F K_{b}+4 \kappa T^{o} \tau /\left(R_{l} e^{2} g^{2}\right) .
$$

\section{ERROR RATE ANALYSIS}

\section{A. Symbol and Bit Error Rate for Single-Pulse PPM}

Consider a PPM mark with $K_{s}$ signal photons and thermal noise. The APD performs a threshold detection, whose probability of miss-detecting a mark can be characterized by the conventional Gaussian tail function [13]. Specifically, given the instantaneous SNR $\Gamma\left(K_{s}\right)$, the (conditional) probability of misdetecting a PPM mark (i.e. missed detection) is given by

$$
\begin{aligned}
P_{e}\left(K_{s}\right) & =Q\left(\sqrt{\Gamma\left(K_{s}\right)}\right), \\
& =\frac{1}{\pi} \int_{0}^{\pi / 2} \exp \left(-\frac{K_{s}^{2}}{2\left(F K_{s}+K_{n}\right) \sin ^{2}(\theta)}\right) d \theta .
\end{aligned}
$$

where $Q(\cdot)$ is the Gaussian-Q function defined as:

$$
\begin{aligned}
Q(x) & =\frac{1}{\sqrt{2 \pi}} \int_{x}^{\infty} e^{-t^{2} / 2} d t, \\
& =\frac{1}{\pi} \int_{0}^{\pi / 2} e^{-x^{2} /\left(2 \sin ^{2}(\theta)\right)} d \theta .
\end{aligned}
$$

Because of the atmospheric scintillation, the photon count per PPM mark $K_{s}$ follows some fading distribution. Specifically, under strong atmospheric scintillations, $K_{s}$ follows the negative exponential distribution as shown in (1), and the probability of misdetecting a PPM mark can be evaluated by averaging $P_{e}\left(K_{s}\right)$ over $K_{s}$ :

$$
P_{e}=\int_{0}^{\infty} P_{e}\left(K_{s}\right) f\left(K_{s}\right) d K_{s} .
$$

Substituting (5) and (1) in (8), we get

$$
P_{e}=\frac{1}{\lambda_{s} \pi} \int_{0}^{\pi / 2} \underbrace{\int_{0}^{\infty} \exp \left(\frac{-K_{s}^{2}}{2\left(F K_{s}+K_{n}\right) \sin ^{2} \theta}-\frac{K_{s}}{\lambda_{s}}\right) d K_{s}}_{A(\theta)} d \theta
$$

Let $x \triangleq F K_{s}+K_{n}$, we can simplify $A(\theta)$ to

$$
\begin{aligned}
A(\theta)= & \frac{1}{F} \int_{0}^{\infty} \exp \left(\frac{\left(x-K_{n}\right)^{2}}{2 F^{2} \sin ^{2} \theta x}-\frac{x-K_{n}}{F \lambda_{s}}\right) d x, \\
= & \frac{1}{F} \exp \left(\frac{K_{n}}{F}\left(\frac{1}{\lambda_{s}}+\frac{1}{F \sin ^{2} \theta}\right)\right) \\
& \int_{0}^{\infty} \exp \left(\frac{x}{F \lambda_{s}}\right) \exp \left(-a x-b \frac{1}{x}\right) d x,
\end{aligned}
$$

where $a=-\frac{1}{2 F^{2} \sin ^{2} \theta}$, and $b=-\frac{K_{n}^{2}}{2 F^{2} \sin ^{2} \theta}$.

Using the Taylor expansion $\exp \left(-\frac{x}{F \lambda_{s}}\right)=$ $\sum_{v=0}^{\infty} \frac{1}{v !}\left(\frac{-x}{F \lambda_{s}}\right)^{v}$, the expression in (11) becomes

$$
\begin{aligned}
A(\theta)= & \frac{1}{F} \exp \left(\frac{K_{n}}{F}\left(\frac{1}{\lambda_{s}}+\frac{1}{F \sin ^{2} \theta}\right)\right) \\
& \sum_{v=0}^{\infty} \frac{(-1)^{v}}{v !\left(F \lambda_{s}\right)^{v}}\left[\int_{0}^{\infty} x^{v} \exp \left(-a x-b \frac{1}{x}\right) d x\right] .
\end{aligned}
$$

Using the following equality [14],

$$
\int_{0}^{\infty} x^{v} \exp \left(-a x-b \frac{1}{x}\right) d x=2\left(\frac{b}{a}\right)^{(v+1) / 2} \mathbf{K}_{v+1}(2 \sqrt{a b}),
$$

where $\mathbf{K}_{v+1}(\cdot)$ is the modified Bessel function of the second kind of order $(v+1)$, we get

$$
\begin{aligned}
A(\theta)= & \frac{2}{F K_{n}} \exp \left(\frac{K_{n}}{F}\left(\frac{1}{\lambda_{s}}+\frac{1}{F \sin ^{2} \theta}\right)\right) \\
& \sum_{v=0}^{\infty} \frac{1}{v !}\left(-\frac{K_{n}}{F \lambda_{s}}\right)^{v} \mathbf{K}_{v+1}\left(\frac{K_{n}}{F^{2} \sin ^{2} \theta}\right) .
\end{aligned}
$$


Inserting (14) into (9), we get the symbol error probability of $M$-slot PPM:

$P_{e}=\frac{2 \beta e^{\beta}}{K_{n}^{2} \pi} \sum_{v=0}^{\infty}\left[\frac{1}{v !}(-\beta)^{v} \int_{0}^{\pi / 2} \exp (B(\theta)) \mathbf{K}_{v+1}(B(\theta)) d \theta\right]$,

where $\beta=\frac{K_{n}}{F \lambda_{s}}$ and $B(\theta)=\frac{K_{n}}{F^{2} \sin ^{2} \theta}$.

In the case of negligible background radiation (known in the optical jargon as the quantum-limited case), we have $K_{n}=0$. For high data-rate free-space optical communication under the negative exponential fading, large $K_{s}$ are typically used to achieve a low probability of error. In such cases, it is reasonable to assume zero or nearly-zero background radiation/noise at the cost of a reduced effective signal strength [10]. The symbol error rate $P_{e}$ in (8) can then be significantly simplified to [10]

$$
\text { Quantum-limited: } \begin{aligned}
P_{e} & =\frac{1}{\pi} \int_{0}^{\pi / 2} \frac{\sin ^{2} \theta}{\frac{K_{s}}{2 F}+\sin ^{2} \theta} d \theta, \\
& =\frac{1}{2}\left(1-\sqrt{\frac{K_{s}}{K_{s}+2 F}}\right) .
\end{aligned}
$$

It is also worth noting that, in the quantum-limited case, photons counts (above the detection threshold) can only occur in the mark slot, such that the probability of detecting nonzero photon counts in the space slot is zero. In other words, the APD detection error is highly asymmetric with a probability $P_{e}$ for misdetecting a mark, but zero probability of misdetecting a space. As shown in Fig. 1, the FSO channel therefore acts like a Z-channel with a mark-to-space cross-over probability of $q=P_{e}$. In general, for a properly-tuned practical FSO system, even though there may be non-zero probability of misdetecting a space, the probability of this type of error is usually magnitudes lower than the probability of misdetecting a mark, such that it can be safely ignored.

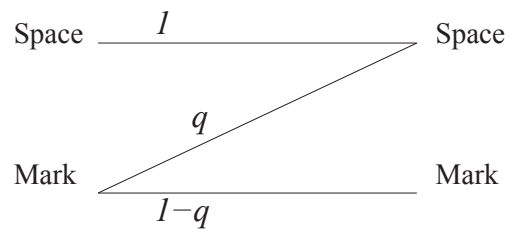

Fig. 1. Z-channel model for PPM FSO communications in the quantumlimited case, where the asymmetric cross-over probability $q=P_{e}$.

To compute the bit error rate, we consider the ensemble average scenario, where the labeling for the PPM symbol is arbitrary, and a misdetection could cause the demodulator to output a random PPM symbol. As such, the bit error rate of the $M=2^{m}$-slot PPM takes the form of:

$$
\begin{aligned}
P_{b} & =\frac{\sum_{k=1}^{m} k\left(\begin{array}{c}
m \\
k
\end{array}\right)}{m 2^{m}} P_{e}, \\
& \approx P_{e} / 2 .
\end{aligned}
$$

\section{B. Symbol and Bit Error Rate for Dual-Pulse PPM}

In the dual-pulse PPM case, a symbol error occurs as soon as any one of the two marks is misdetected. Let $P_{e}$ be the error probability of misdetecting a mark in an $M$-slot PPM. Since the probability of misdetecting a space is negligible compared to $P_{e}$, we have the following expression for the dual-pulse PPM SER:

$$
P_{e}^{d p}=1-\left(1-P_{e}\right)^{2}=2 P_{e}-P_{e}^{2},
$$

where the superscript "dp" stands for dual-pulse.

We now evaluate the bit error rate for dual-pulse PPM systems. Consider $M=2^{m}$ PPM slots. There exist $M(M-$ 1) $/ 2=2^{m-1}\left(2^{m}-1\right)$ possible dual-pulse symbols, and hence can convey $\left\lfloor\log _{2}\left(2^{m-1}\left(2^{m}-1\right)\right)\right\rfloor=2 m-2$ bits per dualpulse symbol. Clearly, the bit error rate of a dual-pulse PPM modulation relates to the actual labeling scheme. We consider two classes of dual-pulse labeling schemes:

- Class I: The set of $2^{2 m-2}$ valid dual-pulses are randomly selected, and each dual-pulse combination is randomly but uniquely mapped to a length- $(2 m-2)$ bit sequence. Such a dual-pulse modulation scheme represents the ensemble average performance of all the dual-pulse PPM schemes. An example of $M=16$ is shown in Fig. 2(a).

- Class II: Class II labeling is a subset of Class I labeling, by setting the constraint that one pulse must be picked from, for example, the first $M / 2$ half slots, and the other must be picked from the second $M / 2$ half slots. Each pulse is separately labeled with $\log _{2}(M / 2)=m-1$ bits, so that the two pulses combined lead to an $m$-bit sequence. The result is like two $M / 2$-slot single-pulse PPM symbols concatenated; see Fig. 2. This type of labeling scheme would in general make the mapping table less complex (than the more general case in Type I), as well as reduce the number of bit errors (in the presence of a symbol error).

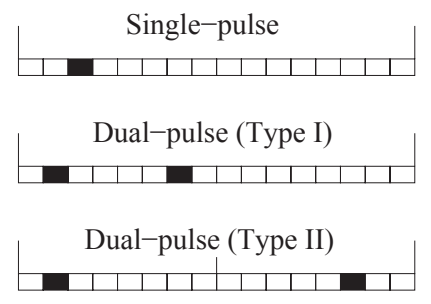

Fig. 2. Single-pulse PPM vs dual-pulse PPM. ( $M=16$ slots per symbol $)$

Class I: Due to the random selection of dual-pulses and their random labels, if a dual-pulse symbol errs, it will incur, on average, the same number of erroneous bits, regardless of the detection error involves only one mark or both marks. The average bit error rate can be computed by

$$
\begin{aligned}
P_{b}^{d p} & =\frac{\sum_{k=1}^{2 m-2} k\left(\begin{array}{c}
2 m-2 \\
k
\end{array}\right)}{(2 m-2) 2^{2 m-2}} P_{e}^{d p}, \\
& \approx P_{e}-P_{e}^{2} / 2 .
\end{aligned}
$$


Class II: Since each mark specifies $(m-1)$ bits, independent of the other, there is a difference between the one-mark error and the two-mark error. The average number of bit errors induced by an arbitrary one-mark error (with the other mark correctly detected) is $\sum_{k=1}^{m-1} k\left(\begin{array}{c}m-1 \\ k\end{array}\right) / 2^{m-1}$, If both marks err, then the number of bit errors gets doubled. Combining these cases, we obtain the average bit error rate of Class-II dualpulse PPM

$P_{b}^{d p}=2 P_{e}\left(1-P_{e}\right) \frac{\sum_{k=1}^{m-1} k\left(\begin{array}{c}m-1 \\ k\end{array}\right)}{(2 m-2)\left(2^{m-1}\right)}+P_{e}^{2} \frac{2 \sum_{k=1}^{m-1} k\left(\begin{array}{c}m-1 \\ k\end{array}\right)}{(2 m-2)\left(2^{m-1}\right)}$,

$$
\approx P_{e}^{2} / 2+P_{e}\left(2-P_{e}\right) / 2=P_{e} / 2 .
$$

It should be noted that the SER and BER expressions for the $M=2^{m}$-slot dual-pulse PPM in (20), (22) and (24) are evaluated using the same mark energy (i.e $K_{s}$ photons per mark) as the single-pulse PPM. Since dual-pulse PPM has two marks per $M$-slot symbol and carries $(2 m-2)$ bits/symbol, when comparing it to single-pulse PPM that has one mark and carries $m$ bits/symbol, an energy penalty of $m /(m-1)$ must be added to the dual-pulse PPM.

\section{Capacity of FSO Channels}

Before numerically assessing the performance of singlepulse and dual-pulse PPM, let us analyze the capacity of the Z-channel FSO communication systems depicted in Fig. 1.

Since the Z-channel is not a symmetric channel, the optimal binary input distribution is therefore non-uniform. Let the input $X$ be a general Bernoulli distribution with the probability of mark (" 1 ") being $\alpha$. The output $Y$ also follows a Bernoulli distribution with the probability of mark being $\alpha(1-q)$. Following the definition of the capacity, we get

$$
\begin{aligned}
\mathcal{C}_{z}(q) & =\max _{\alpha} I(X ; Y)=\max _{\alpha}(H(Y)-H(Y \mid X)), \\
& =\max _{\alpha}(H(\alpha(1-q))-\alpha H(q)),
\end{aligned}
$$

where $q \triangleq \operatorname{Pr}(Y=0 \mid X=1)=P_{e}$ is the crossover probability, and $H(x)=-x \log _{2} x-(1-x) \log _{2}(1-x)$ is the binary entropy function.

With some simplification, we can show that the maximum value of $\mathcal{C}_{Z}(q)$ is given by

$$
\begin{aligned}
\mathcal{C}_{z}(q) & =H\left(\frac{1}{1+2^{H(q) /(1-q)}}\right)-\frac{H(q) /(1-q)}{1+2^{H(q) /(1-q)}}, \\
& =\log _{2}\left(1+(1-q) q^{q /(1-q)}\right)
\end{aligned}
$$

which is attained for $\alpha=\frac{1 /(1-q)}{1+2^{H(q) /(1-q)}}$. For very small $q$, the capacity can be well approximated to

$$
\mathcal{C}_{z}(q) \approx 1-H(q) / 2 .
$$

\section{Single-Pulse vs Dual-Pulse PPM}

To establish the true value of DPPM, we now provide a multi-facet comparison between single-pulse and dual-pulse PPM. We are aware of a couple of previous studies, which,
TABLE I

INFORMATION-THEORETIC PAYLOAD FOR $l$-PULSE PPM

\begin{tabular}{|c|c|c|c|c|c|c|}
\hline & \multicolumn{2}{|c|}{$M=8$} & \multicolumn{2}{c|}{$M=16$} & \multicolumn{2}{c|}{$M=32$} \\
\hline$l$ & \# of sym & bit/sym & \# of sym & bit/sym & \# of sym & bit/sym \\
\hline 1 & 8 & 3 & 16 & 4 & 32 & 5 \\
\hline 2 & 28 & 4.81 & 120 & 6.91 & 496 & 8.95 \\
\hline 3 & 56 & 4.81 & 560 & 9.13 & 4960 & 12.28 \\
\hline 4 & 70 & 6.13 & 1820 & 10.83 & 35960 & 15.13 \\
\hline 5 & & & 4368 & 12.09 & 201376 & 17.62 \\
\hline 6 & & & 8008 & 12.97 & 906192 & 19.79 \\
\hline
\end{tabular}

coming from fairly idealistic premises, show that going dualpulses does not really provide additional benefits over singlepulse (e.g. [4]). Our assessment below will consider both ideal and practical scenarios.

From the information-theoretic perspective, an $M$-slot PPM can support $M$ single-pulse symbols, up to $\left(\begin{array}{c}M \\ 2\end{array}\right)$ dual-pulse symbols, and up to $\left(\begin{array}{c}M \\ l\end{array}\right) l$-pulse symbols. Clearly, as long as $l$ does not increase beyond $M / 2$, the larger the value of $l$, the larger the number of $l$-pulse symbols that are available, and the larger the payload there is to deliver (measured in terms of the number of information bits per modulation symbol). This speaks in favor of multi-pulse (many-pulse) PPM, and the gain in the payload can be quite substantial especially for large $M$. For example, Table I lists the number of available symbols and the payload for $M=8,16,32$ and $l=1,2,3,4,5,6$. As we can see, with $M=32$, single-pulse PPM can carry 5 bits per symbol, whereas quad-pulse PPM can carry three times more bits per symbol, and 6-pulse PPM can carry close to four times as many bits per symbol.

It should be noted that the higher information-theoretic payload (for the same $M$ ) comes at the cost of a (considerably) higher complexity as well as a (small) performance degradation. An $l$-pulse PPM symbol has roughly $l$ times as high as a detection error as that of a single-pulse symbol. For a fair evaluation between single-pulse and dual-pulse PPM, we plot their error rate performance for different values of $M$, in Figures 3-6. In all of the cases, APD noise factor is set to $F=9$, and the $\mathrm{X}$-axis denotes the normalized energy (i.e. photon count per information bit), measured in $\mathrm{dB}$.

Fig. 3 demonstrates the BER and the SER for single-pulse PPM, and Fig. 4 does dual-pulse PPM. In either case, BER is slightly better than the respective SER, and for dual-pulse PPM, Class-II BER is slightly better than that of the general ensemble average (i.e. Class-I). It should be noted that, as $M$ increases, although the error rate performance also improves, the gain is very small and practically negligible for high $M$. Hence, it is fair to say that increasing $M$ brings in only marginal energy efficiency.

Now comparing the SER and the BER curves between single-pulse PPM and dual-pulse PPM in Fig. 5 and Fig. 6, we observe that, for the same value of $M$, (i) dual-pulse incurs a slightly higher SER than single-pulse; and (ii) the average BER of Class-II dual-pulse PPM is extremely close to that of single-pulse PPM, and the average BER of Class-I dual-pulse PPM is slightly higher. 


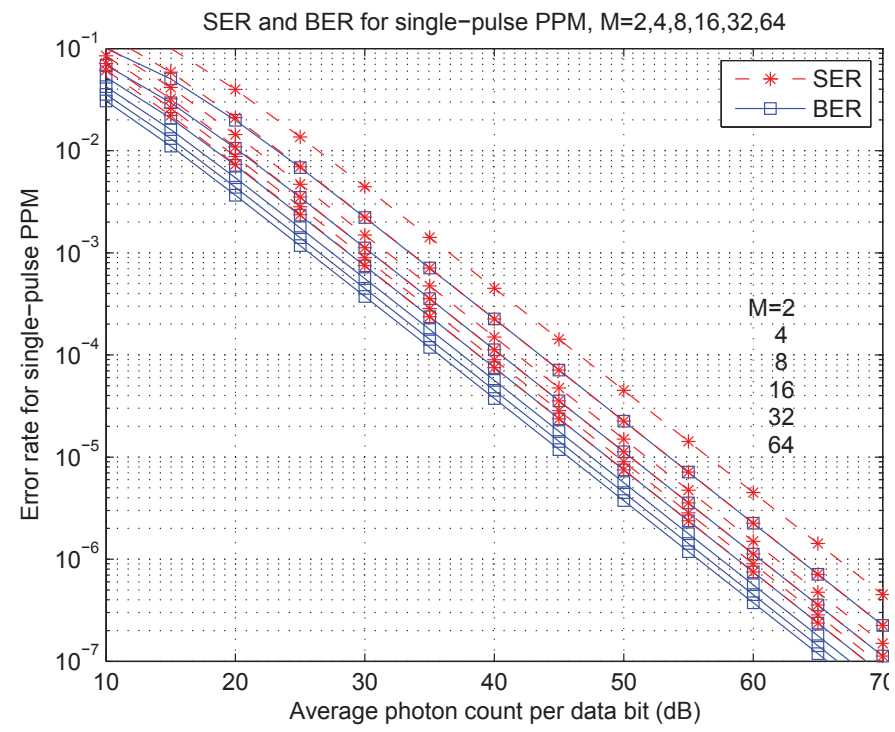

Fig. 3. SER (red lines) and BER (blue lines) for single-pulse PPM. From top down: $M=2,4,8,16,32,64$.

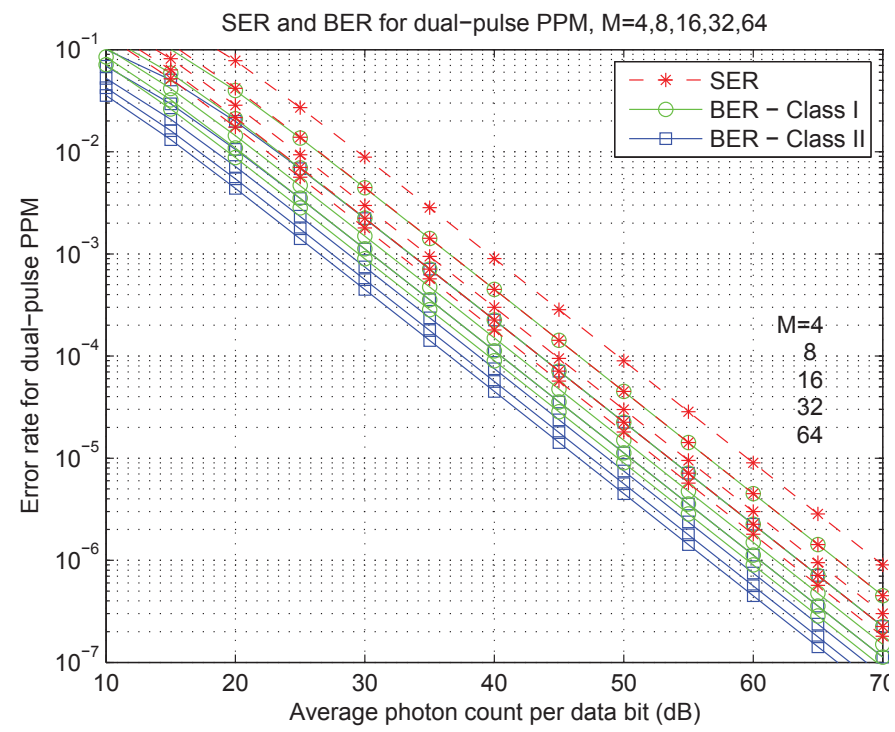

Fig. 4. SER (red) and BER (blue for Class-I, and green for Class-II) for dual-pulse PPM. From top down: $M=4,8,16,32,64$.

It is then tempting to conclude that, disregard the higher complexity, dual-pulse PPM is advantageous over singlepulse PPM in that it provides a considerably higher payload with a minor performance degradation. However, on second thought, the comparison has not been entirely fair. We caution that unlike phase shift keying (PSK) and quadratic amplitude modulation (QAM), where a higher modulation order implies a more efficient bandwidth utilization (measured in $\mathrm{bit} / \mathrm{Hz} / \mathrm{second}$ ), increasing the order of PPM also cause an increase in the symbol interval (for the same slot width $\tau$ ) and hence a reduced bit/Hz/second. For example, 8-slot singlepulse PPM with slot width $\tau$ (seconds) and frequency band $B$ $(\mathrm{Hz})$, delivers a bandwidth efficiency of $\frac{3}{8} \frac{1}{B \tau} \mathrm{bit} / \mathrm{Hz} / \mathrm{s}$, whereas going to $M=16$ reduces the bandwidth efficiency to $\frac{1}{4} \frac{1}{B \tau}$ $\mathrm{bit} / \mathrm{Hz} / \mathrm{s}$, and $M=32$ further reduces it to $\frac{5}{32} \frac{1}{B \tau} \mathrm{bit} / \mathrm{Hz} / \mathrm{s}$. Hence, for single-pulse PPM, the most bandwidth efficient

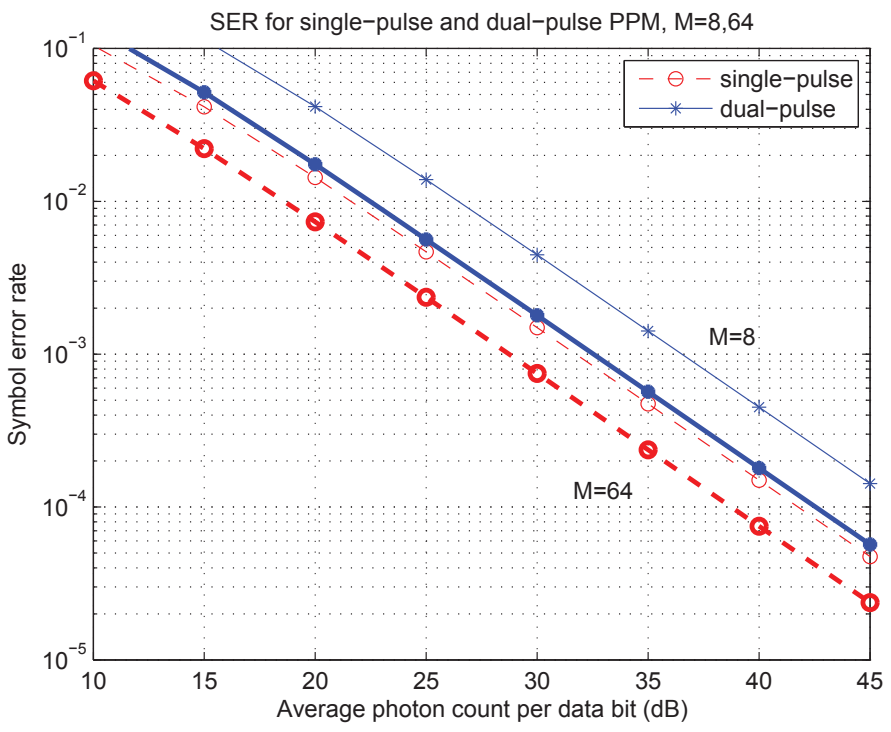

Fig. 5. SER comparison between dual-pulse (blue) and single-pulse (red) PPM. From top down: $M=8,64$.

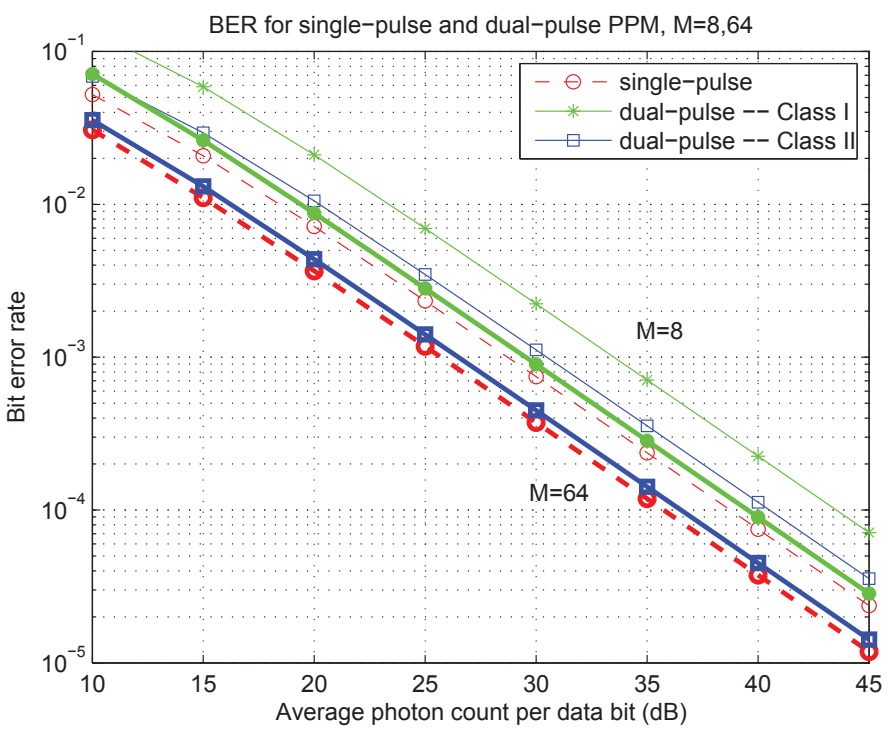

Fig. 6. BER comparison between dual-pulse (green for Class-I, blue for Class-II) and single-pulse (red) PPM. From top down: $M=8,64$.

case is $M=2$ or 4 , both delivering $\frac{1}{2} \frac{1}{B \tau} \mathrm{bit} / \mathrm{Hz} / \mathrm{s}$. Now consider the dual-pulse case. From Fig. 2 and all the performance curves, Type-II $M$-slot dual-pulse PPM is essentially $M / 2$-slot single-pulse PPM, where two symbols of the latter concatenatively form one symbol of the former. Since the more general dual-pulse case (i.e. Class-I) is even worse than ClassII performance-wise and complexity-wise, this leads to the following immediate implications: first, there is no gain to go from single-pulse to dual-pulse; and second, there is no need to go for large $M$, and the 4-slot single-pulse PPM is both simple and most bandwidth efficient.

To see this, Fig. 7 evaluates the "capacity" of different PPM schemes. The X-axis denotes the power efficiency, measured in photon count per information bit $(\mathrm{dB})$, and the Y-axis denotes the bandwidth efficiency, measured in normalized 
bit/Hz/second. The capacity of the Z-channel for both the optimal input distribution and the uniform input distribution are shown. Since here the crossover probability $q$ is the probability of misdetecting a mark, which is upper bounded by 0.5 (i.e. random guess), the capacity can never drop to 0 . In fact, for optimal input, the limit is $C_{z}(0.5)=0.3219 \mathrm{bit} / \mathrm{Hz} / \mathrm{s}$, and for uniform input, that is $0.3113 \mathrm{bit} / \mathrm{Hz} / \mathrm{s}$. The PPM systems are evaluated at BER of $10^{-5}$, with uniform input. We see that as $M$ increases, the energy-efficiency increases slightly, but the bandwidth-efficiency considerably decreases. This clearly speaks for the preference to stay with low $M$, and 4-slot single-pulse PPM, or, 8-slot dual-pulse PPM, strikes the best balance between bandwidth and energy efficiency.

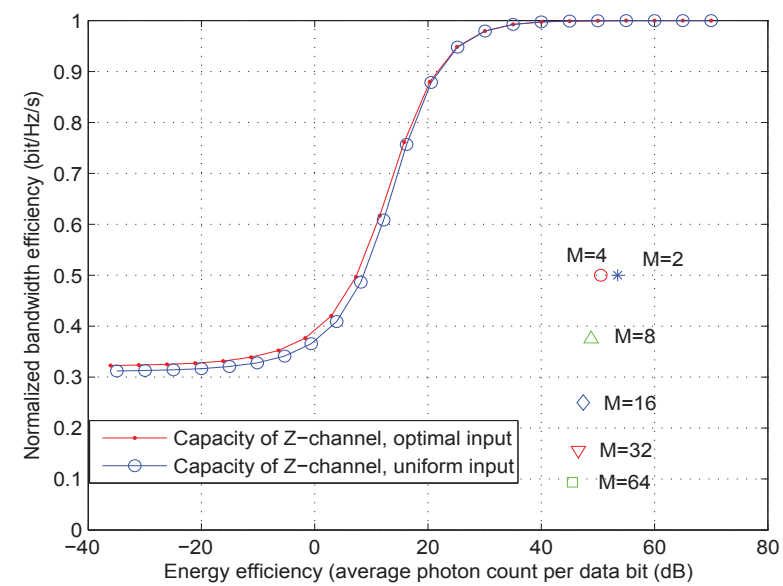

Fig. 7. Bandwidth efficiency vs energy efficiency for singel-pulse PPM.

The previous conclusions would be all valid if there is no requirement for guard time $T_{g}$. In practice, many laser emitting systems have a pulse repetition frequency (PRF) constraint, which specifies how frequently two laser pulses (of a decent strength) can be generated, and which translates to the requirement for a minimum and a maximum time separation between two pulses. This practicality issue immediately changes the picture in two ways. First, in the case of single-pulse PPM, a very small $M$ would result in a very low channel utilization, as a considerable amount of time is spent to guard between two very short symbols. Hence, an appropriate value of $M$ should be selected in order to deliver the best throughput under a given PRF constraint. Second, now there is benefit to be gained by going from $(M / 2)$-slot single-pulse PPM to $M$ slot dual-pulse PPM. As shown in Fig. 8, with the appropriate system specifications, there exists an adequate pool of dualpulses meeting the separation requirement, such that explicit guard time is needed only after every $M$ slots. For example, for $M=8$, it is possible to design dual-pulse PPM with a minimum of $12.5 \%$ separation between the two pulses (i.e. 1 slot separation); and for $M \geq 16$, dual-pulse PPM with a minimum of $25 \%$ separation (i.e. $M / 4$ slots) is attainable between the two pulses. On the other hand, single-pulse PPM would need guard time after every $M / 2$ slots. Clearly, dualpulse enhances the channel utilization and hence the data throughput. When there is substantial guard time constraint, such as a minimum of 16 slots, then dual-pulse PPM can promise to be $20 \%$ more bandwidth efficient than single-pulse PPM, while delivering the same energy efficiency.

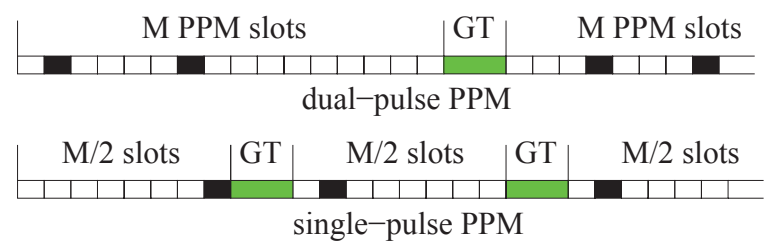

Fig. 8. Illustration of guard time (GT) and channel utilization for single-pulse and dual-pulse PPM.

\section{CONClusion}

We have evaluated the error rate performance and the bandwidth effiency for dual-pulse PPM as well as for singlepulse PPM under strong turbulent channels. We show that without any guard time constraint, there is no benefit of going from single-pulse to dual-pulse, and that 4-slot singlePPM strikes the best trade-off between energy efficiency and bandwidth efficiency. When there is substantial guard time constraint, then dual-pulse PPM can effectively improve the channel utilization and hence the system throughput.

\section{REFERENCES}

[1] H. Sugiyama and K. Nosu, "MPPM: A method of improving the bandutilization efficiency in optical PPM," J of Lightwave Tech., vol. 7. no. 3, pp. 465-472. March 1999.

[2] J. M. Budinger, M. J. Vanderaar, "Combinatorial pulse position modulation for power-efficient free-space laser communications," Free-Space Laser Communication Technologies V, SPIE Vol. 1866, pp. 214-225, Jan 1993.

[3] A. D. Wyner, "Capacity and error exponent for the direct detection photon channel-Part I," IEEE Trans on Inf. Theory, vol. 34, pp. 14491461, Nov. 1988.

[4] J. Hamkins and B. Moisions, "Multipulse pulse-position modulation on discrete memoryless channels," The Interplanetary Network Progress Report, vol. 42-161, Jet Propulsion Laboratory, pp. 1-13, May 15, 2005.

[5] A. B. Siddique, and M. Tahir, "Joint brightness control and data transmission for visible light communication systems based on white LEDs," Proc. of 8th IEEE Consumer Commun. and Networking Conf., 2011.

[6] M. Uysal and J. Li, "Error performance analysis of coded wireless optical links over atmospheric turbulence channels," IEEE Wireless Communication and Networking Conf (WCNC),, vol. 4, pp. 2405-2410, 2004.

[7] L. C. Andrews, R. L. Philips, and C. Y. Hopen, Laser Beam Scintillation with Applications, SPIE Press, 2001

[8] M. Uysal and J. Li, "Error rate performance of coded free-space optical links over gamma-gamma turbulence," IEEE Intl. Conf. Commun. (ICC), pp. 3331-3335, 2004.

[9] T. A. Tsiftsis, "Performance of heterodyne wireless optical communication systems over gamma-gamma atmospheric turbulence channels," Electronics Letters, pp. 372-373, 2008.

[10] K. Kiasaleh, "Performance of APD-based, PPM free-space optical communication systems in atmospheric turbulence," IEEE Trans Commun., vol 53, no. 9, Sept, 2005, pp. 1455-1461

[11] J. Anguita, I. Djordjevic, M. Neifeld, and B. Vasic, "Shannon capacities and error-correction codes for optical atmospheric turbulent channels," Journal of Optical Networking, Vol. 4, Issue 9, pp. 586-601, 2005

[12] J. Singh1, and V. K. Jain, "Performance Analysis of BPPM and $M$ ary PPM Optical Communication Systems in Atmospheric Turbulence," IETE Technical Review, pp. 146-153, 2008

[13] R. M. Gagliardi and S. Karp, Optical Communications, New York: Wiley, 1995.

[14] I. S. Gradshteyn, and I. M. Ryzhik, Table of Integrals, Series and products, Academic Press, 1994. 


